\section{GIST: Nilotinib nicht besser als Imatinib}

Imatinib ist der Standard in der Erstlinientherapie fortgeschrittener gastrointestinaler Stromatumoren (GIST). Nun gibt es auch Daten zu Nilotinib in der Erstlinie.

$\checkmark$ on einer Therapie mit dem Tyrosinkinaseinhibitor (TKI) Imatinib profitieren besonders GIST-Patienten mit aktivierender Mutation im Exon 11 des KIT-Gens, während die Ergebnisse bei Exon-9- und PDGFRa-Mutation oder bei KIT- und PDGFR $a$-Wildtyp oft wesentlich schlechter sind. Nilotinib ist ein TKI der zweiten Generation, der in vitro auch einige KIT-Mutationen hemmen konnte, die gegenüber Imatinib resistent waren. Im Rahmen der Phase-III-Studie ENESTg1 wurde daher untersucht, ob Nilotinib in der Erstlinie zu besseren Ergebnissen führt als Imatinib.

Aufgenommen wurden Patienten mit inoperablem und/oder metastasiertem GIST. Sie erhielten entweder täglich Ima- tinib (1-mal $400 \mathrm{mg}$ oder KIT-Exon9-Mutation: 2-mal $400 \mathrm{mg}$ ) oder Nilotinib (2-mal $400 \mathrm{mg}$ ). Bei Progress war ein Wechsel in den anderen Arm vorgesehen. Primäres Studienziel war das progressionsfreie Überleben (PFS).

In der ersten geplanten Interimsanalyse nach 75 Fällen mit Progress $(\mathrm{n}=397)$ zeigte sich, dass unter Nilotinib deutlich mehr Patienten progredient waren als unter Imatinib (24 vs. $14 \%$; Hazard Ratio [HR] 2,03). In den Nilotinib-Arm wurden daraufhin keine Patienten mehr rekrutiert. Ein Wechsel von Imatinib zu Nilotinib war nicht mehr möglich, umgekehrt konnten die Nilotinib-Patienten auch ohne Progressionsnachweis zu Imatinib wechseln.

In der finalen Analyse $(\mathrm{n}=644)$ war die Rate für das 2-Jahres-PFS unter Imatinib mit 59,2\% höher als unter Nilotinib mit 51,6\% (HR 1,47, $95 \%$-Konfidenzintervall [95\%-KI] 1,1-1,95). Auch die Rate für das 2-Jahres-Überleben insge- samt war im Imatinib-Arm höher (90,0 vs. $81,8 \%$; HR $1,85,95 \%$-KI 1,2-2,86). Das Grad-3/4-Nebenwirkungsprofil war in beiden Gruppen ähnlich.

Bei Patienten mit KIT-Exon-9-Mutation waren PFS und Überleben nach 24 Monaten bei niedrigen Fallzahlen $(\mathrm{n}=50)$ unter Imatinib höher als unter Nilotinib. Bei Exon-11-Mutation $(\mathrm{n}=266)$ fanden sich hinsichtlich des PFS keine Unterschiede, wohl aber in der Überlebensrate, die unter Imatinib mit 96,2 \% höher war als unter Nilotinib mit 87,5\% (HR 3,00, $95 \%$-KI 1,16-7,74).

Fazit: Nilotinib kann nicht für die Erstlinie bei Patienten mit inoperablem und/ oder metastasiertem GIST empfohlen werden. Möglicherweise lassen sich in Zukunft Subgruppen von GIST-Patienten identifizieren, für die Nilotinib initial von Nutzen ist.

Brigitte Schalhorn

Blay JY et al. Nilotinib versus imatinib as firstline therapy for patients with unresectable or metastatic gastrointestinal stromal tumours (ENESTg1): a randomised phase 3 trial. Lancet Oncol. 2015;16(5):550-60.

\title{
Pankreaskarzinom: länger leben durch adjuvante Chemoradiotherapie
}

\begin{abstract}
Welchen Nutzen hat die Addition der Radiotherapie zur heute allgemein akzeptierten postoperativen Chemotherapie bei Patienten mit einem Adenokarzinom des Pankreas. Dieser Frage gingen US-amerikanische Forscher nach.
\end{abstract}

$\mathrm{D}$ as Team um Charles E. Rutter analysierte die Überlebensdaten aller Patienten der US-amerikanischen National Cancer Data Base (NCDB), die zwischen 1998 und 2009 die Diagnose Adenokarzinom des Pankreas erhalten hatten (Stadium pT1-3 N0-1 M0, Tumor R0oder R1-reseziert) und adjuvant mit einer Chemotherapie (CT) oder Radiochemotherapie (RCT) behandelt worden waren. Von den 6.165 Patienten hatten 2.334 $(38 \%)$ eine adjuvante CT erhalten und 3.831 (62\%) eine RCT.

Sowohl in der uni- als auch in der multivariaten Analyse verschlechterte sich die Prognose mit zunehmendem T-Stadium, bei N1-Lymphknotenbefall (vs. N0) und nach R1-Resektion (vs. R0) signifikant. Gegenüber der alleinigen CT führte die adjuvante RCT in der univariaten Analyse zu einem signifikanten Anstieg im medianen Überleben (22,3 vs. 20,0 Monate; $p=0,001$ ), zu einer höheren 5-Jahres-Überlebensrate (19,6 vs. 16,5\%; $\mathrm{p}<0,001)$ und in der multivariaten Analyse zu einer Risikoreduktion für Tod jeglicher Ursache (Hazard Ratio [HR] 0,893; $\mathrm{p}=0,001)$. Diese Risikoreduktion blieb auch bestehen, wenn man gematchte CT- und RCT-Patientengruppen verglich. Im Rahmen der RCT führten sowohl eine Mono- als auch eine Kombinationschemotherapie zu einer signifikanten Senkung des Mortalitätsrisikos.

Die Subgruppenanalyse bestätigte das im Vergleich zur alleinigen CT bessere Überleben nach RCT besonders bei Patienten mit pT3 (HR 0,892; p = 0,003) und bei N1-Lymphknotenbefall (HR
0,856; $\mathrm{p}<0,001)$. Die positiven Effekte zeigten sich auch bei R0- und R1-Resektion. In den frühen Stadien pT1 und pT2 sowie bei negativem Lymphknotenstatus (pN0) wurde der Therapieerfolg durch die zusätzliche Radiotherapie dagegen nicht verbessert.

Fazit: Nach R0- und R1-Resektion eines Adenokarzinoms des Pankreas verlängerte die adjuvante RCT in einer großen, nationalen Patientenkohorte das Überleben im Vergleich zur alleinigen CT. Besonders Patienten mit N1-Lymphknotenstatus und/oder R1-Resektion profitierten von der kombinierten Therapie. Zum endgültigen Beweis sind allerdings randomisierte Studien erforderlich.

Brigitte Schalhorn

Rutter CE et al. Addition of radiotherapy to adjuvant chemotherapy is associated with improved overall survival in resected pancreatic adenocarcinoma: An analysis of the National Cancer Data Base. Cancer. 2015;121(23):4141-9. 